\title{
Palladium-Catalyzed Amination in the Synthesis of Macrocycles Comprising Two Naphthalene And Two Polyamine Moieties
}

\author{
Alexei N. Uglov, ${ }^{a}$ Alexei D. Averin, ${ }^{\mathrm{a}, \mathrm{b} @}$ Alexei K. Buryak, ${ }^{\mathrm{b}}$ Alla Bessmertnykh- \\ Lemeune, ${ }^{\mathrm{c}}$ Roger Guilard, ${ }^{\mathrm{c}}$ and Irina P. Beletskaya ${ }^{\mathrm{a}, \mathrm{b}}$ \\ ${ }^{a}$ Lomonosov Moscow State University, Department of Chemistry, 119991 Moscow, Russia \\ ${ }^{\mathrm{b}}$ A.N. Frumkin Institute of Physical and Electrochemistry, 119991 Moscow, Russia \\ 'Institut de Chimie Moléculaire de l'Université de Bourgogne (ICMUB), UMR CNRS 5260, 21078 Dijon Cedex, France \\ ${ }^{\circledR}$ Corresponding authorE-mail: averin@org.chem.msu.ru
}

\begin{abstract}
Two approaches were elaborated for the synthesis of macrocycles comprising two naphthalene and two polyamine moieties (cyclodimers). The first one includes the synthesis of N,N'-bis(7-bromonaphth-2-yl) substituted polyamines via $P d$-catalyzed amination reaction of polyamine with excess of 2,7-dibromonaphthalene, followed by the Pd-catalyzed macrocyclization reaction with appropriate polyamine. The second route comprises the formation of 2,7-bis (polyamine) substituted naphthalenes which are used in situ for the macrocyclization with 2,7-dibromonaphthalene. The yields of cyclodimers are dependent on the nature of polyamines and catalytic systems employed. The two synthetic routes were compared and the one-pot method was found to be advantageous providing better yields of the target products.
\end{abstract}

Keywords: Palladium-catalized amination, naphthalene, polyamines, macrocycles, synthesis.

\section{Палладий-катализируемое аминирование в синтезе макроциклов с Авумя нафталиновыми и Авумя полиаминными фрагментами}

\author{
А. Н. Углов, ${ }^{\text {a }}$ А. А. Аверин, ${ }^{\text {a,b@ }}$ А. К. Буряк, ${ }^{\text {b }}$ А. Бессмертных-лемен, ${ }^{c}$ Р. Гиляр, ${ }^{c}$ \\ И. П. Белецкая ${ }^{\mathrm{a}, \mathrm{b}}$
}

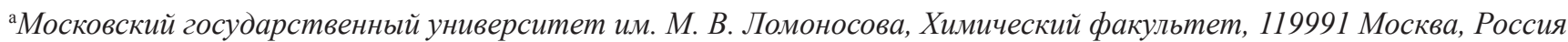
${ }^{\mathrm{b}}$ Институт физической химии и электрохимии им. А. Н. Фрумкина РАН, 119991 Москва, Россия 'Institut de Chimie Moléculaire de l'Université de Bourgogne (ICMUB), UMR CNRS 5260, 21078 Дижон, Франция

${ }^{\circledR}$ E-mail: averin@org.chem.msu.ru

\begin{abstract}
Разработаны два подхода к синтезу макроииклов, содержащих по два фрагмента нафталина и полиамина (циклодимеров). Первый подход заключается в первоначальном получении N,N'-бис(7-бромнафт-2-ил) замещенных полиаминов Pd-катализируемым аминированием избытка 2,7-дибромнафталина полиаминами с последующей Pd-катализируемой макроциклизацией данных соединений с использованием соответствующих полиаминов. Второй подход заключается в синтезе 2,7-бис(полиамино)замещенных нафталинов, которые используются іп situ на второй стадии макроциклизации. Найдено, что выходы макроциклов зависят от природы полиамина, в определенных случаях показано предпочтительное использование фосфиновых лигандов BINAP или Xantphos, произведено сравнение эффективности двух предложенных методов, установлено, что второй метод дает в ичелом более высокие выходы иелевых ичиклодимеров. Выделены и охарактеризованы циклические и линейные побочные продукты в указанных реакциях.
\end{abstract}

Ключевые слова: Палладий-катализируемое аминирование, нафталин, полиамины, макроциклы, синтез. 


\section{Introduction}

Worldwide applications of coordination chemistry in the development of chemotherapeutic or diagnostic agents, treatment of nuclear waste, chemosensoring have become of paramount importance and progress rapidly in comparison with the studies that elucidate the basic theory of this field. Therefore, the research is often carried out in a make-and-try manner and numerous ligands have been synthesized and their affinity to metal cations has been evaluated. Ideally, efficient ligands should match various criteria such as high binding constants and selectivity together with fast binding kinetics of the coordination pocket, water solubility, insensitivity to $\mathrm{pH}$ changes, non-toxicity, etc. Complex molecular architectures should be synthesized to adopt the system properties to a real-life application. Ligand properties could be tuned not only by varying the number and nature of donor groups but also by introducing different non-coordinated structural motifs. Aromatic moieties are of major interest in ligand design for chemosensoring and sequestration of toxic and radioactive metal ions. The first macrocyclic compounds comprising the naphthalene moiety were described in the literature over 70 years ago. ${ }^{[1]}$ This rigid lipophilic fragment possessing interesting photophysical properties draws considerable interest and dozen of works appeared dealing with the synthesis and investigation of the macrocycles of different geometry based on naphthalene, as well with the azacrown ethers derivatives containing naphthalene as exocyclic substituents. In these polyazacycles nitrogen atoms are present in such fragments as Schiff bases, ${ }^{[2]}$ diamides,${ }^{[3]}$ diimides ${ }^{[4]}$ lactames ${ }^{[5]}$ Naphthalene system can be condensed with tetraazamacrocycles, ${ }^{[6]}$ macrocycles may contain phosphorus atoms ${ }^{[7]}$ or only carbon atoms ${ }^{[8]}$ it can be incorporated into calixarene ${ }^{[9]}$ and catenane ${ }^{[10]}$ structures, combined in various manners with porphyrins. [11] Such compounds are valuable molecular precursors for supramolecular studies and could be used e.g. as organic anions receptors ${ }^{[12]}$ or molecular rotors. ${ }^{[13]}$

Recently, we have synthesized a series of the nitrogen- and oxygen-containing macrocycles based on 2,7-diaminonaphthalene (Scheme 1). ${ }^{[14]}$ Herein we report a detailed account on the synthesis of macrocyclic compounds composed by two aromatic groups and two linear chains (socalled cyclodimers).

\section{Experimental}

NMR spectra were registered using Bruker Avance 400 spectrometer, MALDI-TOF spectra were obtained with Bruker Ultraflex spectrometer using 1,8,9-trihydroxyanthracene as matrix and PEGs as internal standards. Dioxa- and trioxadiamines, tetraamine, BINAP and Xantphos ligands, sodium tert-butoxide were purchased from Aldrich and Acros and used without further purification, 2,7-dibromonaphthalene was obtained according to a reported procedure, ${ }^{[15]} \mathrm{Pd}(\mathrm{dba})_{2}$ was synthesized according to the method described. ${ }^{[16]}$ Dioxane was distilled over $\mathrm{NaOH}$ followed by the distillation over sodium under argon, acetonitrile, dichloromethane and methanol were used freshly distilled.

Typical procedure for the synthesis of $N, N^{\prime}$-bis(7bromonaphth-2-yl) substituted naphthalenes 4a-c.

A two-neck flask equipped with a condenser and magnetic stirrer, flushed with dry argon, was charged with 2,7-dibromonaphthalene
2 (629 mg, $2.2 \mathrm{mmol}), \mathrm{Pd}(\mathrm{dba})_{2}(11.5 \mathrm{mg}, 0.02 \mathrm{mmol}, 2 \mathrm{~mol} \%)$ and Xantphos (15 mg, $0.025 \mathrm{mmol}, 2.5 \mathrm{~mol} \%$ ) in the case of dioxa- and trioxadiamines 1a,b, or Pd(dba) (23 mg, $0.04 \mathrm{mmol}, 4 \mathrm{~mol} \%)$ and BINAP (28 mg, $0.045 \mathrm{mmol}, 4.5 \mathrm{~mol} \%$ ) in the case of tetraamine $1 \mathrm{c}, 20$ $\mathrm{ml}$ (in the case of dioxa- and trioxadiamines $\mathbf{1 a}, \mathbf{b}$ ) or $10 \mathrm{ml}$ (in the case of tetraamine 1c) dioxane were added, the mixture was stirred for 2-3 min, then corresponding polyamine $1 \mathbf{a}-\mathbf{c}(1 \mathrm{mmol})$ and ${ }^{t} \mathrm{BuONa}(288$ $\mathrm{mg}, 3 \mathrm{mmol}$ ) were added, and the reaction mixture was refluxed for 5-8 h. After cooling it down to ambient temperature the reaction mixture was diluted with $\mathrm{CH}_{2} \mathrm{Cl}_{2}$, the residue was filtered off, the organic solvents were evaporated in vacuo, and the residue was chromatographed on silica gel using a sequence of eluents: $\mathrm{CH}_{2} \mathrm{Cl}_{2}, \mathrm{CH}_{2} \mathrm{Cl}_{2} / \mathrm{MeOH}$ (200:13:1), $\mathrm{CH}_{2} \mathrm{Cl}_{2} / \mathrm{MeOH} / \mathrm{NH}_{3}$ aq (100:20:1-10:4:1).

$N, N^{\prime}$-(2,2'-(Ethane-1,2-diylbis(oxy))bis(ethane-2,1-diyl)) bis(7-bromonaphthalene-2-amine), $4 \boldsymbol{a}$. Obtained from $148 \mathrm{mg}$ of dioxadiamine 1a. Eluent $\mathrm{CH}_{2} \mathrm{Cl}_{2} / \mathrm{MeOH}$ 500:1-200:1. Yield 150 mg (27\%), yellowish glassy compound. (MALDI-TOF) found: 557.0391. $\mathrm{C}_{26} \mathrm{H}_{27} \mathrm{Br}_{2} \mathrm{~N}_{2} \mathrm{O}_{2}$ requires $557.0439[\mathrm{M}+\mathrm{H}]^{+}$. ${ }^{1} \mathrm{H} \mathrm{NMR}$ $\left(\mathrm{CDCl}_{3}, 298 \mathrm{~K}\right) \delta_{\mathrm{H}} \mathrm{ppm}: 3.38\left(4 \mathrm{H}, \mathrm{t},{ }^{3} J=5.1 \mathrm{~Hz}\right), 3.69(4 \mathrm{H}, \mathrm{s}), 3.77$ $\left(4 \mathrm{H}, \mathrm{t},{ }^{3} J=5.1 \mathrm{~Hz}\right), 4.35(2 \mathrm{H}$, br.s $), 6.66\left(2 \mathrm{H}, \mathrm{d},{ }^{4} J=2.0 \mathrm{~Hz}\right), 6.83$ $\left(2 \mathrm{H}, \mathrm{dd},{ }^{3} J=8.8 \mathrm{~Hz},{ }^{4} J=2.2 \mathrm{~Hz}\right), 7.23\left(2 \mathrm{H}, \mathrm{dd},{ }^{3} J=8.7 \mathrm{~Hz},{ }^{4} J=\right.$ $1.9 \mathrm{~Hz}), 7.47-7.57(4 \mathrm{H}, \mathrm{m}), 7.72$ (2H, br.s). ${ }^{13} \mathrm{C} \mathrm{NMR}\left(\mathrm{CDCl}_{3}, 298\right.$ K) $\delta_{\mathrm{c}} \mathrm{ppm}: 43.3(2 \mathrm{C}), 69.4(2 \mathrm{C}), 70.3(2 \mathrm{C}), 103.4(2 \mathrm{C}), 118.4(2 \mathrm{C})$, 120.5 (2C), 125.2 (2C), 126.0 (2C), 127.8 (2C), 128.9 (2C), 129.3 (2C), $136.4(2 \mathrm{C}), 146.6(2 \mathrm{C})$.

$N, N, N$, '-Tris(7-bromonaphth-2-yl)substituted dioxadiamine $8 \mathbf{8}$ was obtained as the second product in the synthesis of compound 4a. Eluent $\mathrm{CH}_{2} \mathrm{Cl}_{2}$. Yield $50 \mathrm{mg}(10 \%)$, yellowish glassy compound. (MALDI-TOF) found: 761.0087. $\mathrm{C}_{36} \mathrm{H}_{32} \mathrm{Br}_{3} \mathrm{~N}_{2} \mathrm{O}_{2}$ requires 761.0014 $[\mathrm{M}+\mathrm{H}]^{+} .{ }^{1} \mathrm{H} \mathrm{NMR}\left(\mathrm{CDCl}_{3}, 298 \mathrm{~K}\right) \delta_{\mathrm{H}} \mathrm{ppm}: 3.30\left(2 \mathrm{H}, \mathrm{t},{ }^{3} J=5.2 \mathrm{~Hz}\right)$, $3.63(4 \mathrm{H}, \mathrm{s}), 3.70\left(2 \mathrm{H}, \mathrm{t},{ }^{3} J=5.2 \mathrm{~Hz}\right), 3.79\left(2 \mathrm{H}, \mathrm{t},{ }^{3} J=5.9 \mathrm{~Hz}\right), 4.15$ $\left(2 \mathrm{H}, \mathrm{t},{ }^{3} J=5.8 \mathrm{~Hz}\right), 4.26(1 \mathrm{H}, \mathrm{br} . \mathrm{s}), 6.61\left(1 \mathrm{H}, \mathrm{d},{ }^{4} J=1.6 \mathrm{~Hz}\right), 6.74$ $\left(1 \mathrm{H}, \mathrm{dd},{ }^{3} J=8.7 \mathrm{~Hz},{ }^{4} J=2.3 \mathrm{~Hz}\right), 7.26\left(1 \mathrm{H}, \mathrm{dd},{ }^{3} J=9.0 \mathrm{~Hz},{ }^{4} J=1.9\right.$ $\mathrm{Hz}), 7.27\left(2 \mathrm{H}, \mathrm{dd},{ }^{3} J=9.0 \mathrm{~Hz},{ }^{4} J=2.1 \mathrm{~Hz}\right), 7.36\left(2 \mathrm{H}, \mathrm{d},{ }^{4} J=1.8 \mathrm{~Hz}\right)$, $7.39\left(2 \mathrm{H}, \mathrm{dd},{ }^{3} J=8.7 \mathrm{~Hz},{ }^{4} J=1.9 \mathrm{~Hz}\right), 7.48\left(2 \mathrm{H}, \mathrm{d},{ }^{3} J=8.9 \mathrm{~Hz}\right), 7.57$ $\left(2 \mathrm{H}, \mathrm{d},{ }^{3} J=8.7 \mathrm{~Hz}\right), 7.64\left(2 \mathrm{H}, \mathrm{d},{ }^{3} J=9.0 \mathrm{~Hz}\right), 7.72(1 \mathrm{H}, \mathrm{br} . \mathrm{s}), 7.82$ (2H, br.s). ${ }^{13} \mathrm{C} \mathrm{NMR}\left(\mathrm{CDCl}_{3}, 298 \mathrm{~K}\right) \delta_{\mathrm{c}} \mathrm{ppm}: 43.2(1 \mathrm{C}), 52.1(1 \mathrm{C})$, 68.2 (1C), 69.4 (1C), 70.4 (1C), 70.8 (1C), 103.3 (1C), 115.5 (2C), 118.4 (1C), 120.4 (1C), 120.6 (2C), 122.8 (2C), 125.1 (1C), 125.8 (1C), 127.4 (2C), 127.8 (1C), 128.4 (2C), 128.7 (2C), 128.8 (1C), 128.9 (2C), 129.1 (2C), 129.2 (1C), 135.7 (2C), 136.4 (1C), 145.8 (2C), 146.5 (1C).

Oligomer $9 a$ was obtained as the third product in the synthesis of compound 4a. Eluent $\mathrm{CH}_{2} \mathrm{Cl}_{2} / \mathrm{MeOH} 100: 1$. Yield $55 \mathrm{mg}$ (13 $\%$ ), yellowish glassy compound. (MALDI-TOF) found: 829.1922. $\mathrm{C}_{42} \mathrm{H}_{47} \mathrm{Br}_{2} \mathrm{~N}_{4} \mathrm{O}_{4}$ requires $829.1964[\mathrm{M}+\mathrm{H}]^{+}$. ${ }^{1} \mathrm{H}$ NMR $\left(\mathrm{CDCl}_{3}, 298\right.$ K) $\delta_{\mathrm{H}}$ ppm: $3.37\left(8 \mathrm{H}, \mathrm{t},{ }^{3} \mathrm{~J}=5.0 \mathrm{~Hz}\right), 3.67(8 \mathrm{H}, \mathrm{s}), 3.75\left(4 \mathrm{H}, \mathrm{t},{ }^{3} \mathrm{~J}=\right.$ $4.6 \mathrm{~Hz}), 3.76\left(4 \mathrm{H}, \mathrm{t},{ }^{3} J=4.9 \mathrm{~Hz}\right), 4.11(2 \mathrm{H}$, br.s $), 4.37$ (2H, br.s), $6.60\left(2 \mathrm{H}, \mathrm{d},{ }^{3} J=8.0 \mathrm{~Hz}\right), 6.61(2 \mathrm{H}$, br.s $), 6.65\left(2 \mathrm{H}, \mathrm{d},{ }^{4} J=1.9 \mathrm{~Hz}\right)$, $6.83\left(2 \mathrm{H}, \mathrm{dd},{ }^{3} J=8.6 \mathrm{~Hz},{ }^{4} J=2.0 \mathrm{~Hz}\right), 7.23\left(2 \mathrm{H}, \mathrm{dd},{ }^{3} J=8.2 \mathrm{~Hz}\right.$, $\left.{ }^{4} J=1.4 \mathrm{~Hz}\right), 7.40\left(2 \mathrm{H}, \mathrm{d},{ }^{3} J=9.1 \mathrm{~Hz}\right), 7.47\left(2 \mathrm{H}, \mathrm{d},{ }^{3} J=8.3 \mathrm{~Hz}\right)$, $7.50\left(2 \mathrm{H}, \mathrm{d},{ }^{3} \mathrm{~J}=8.7 \mathrm{~Hz}\right), 7.72(2 \mathrm{H}, \mathrm{br} . \mathrm{s}) .{ }^{13} \mathrm{C} \mathrm{NMR}\left(\mathrm{CDCl}_{3}, 298 \mathrm{~K}\right)$ $\delta_{\mathrm{c}} \mathrm{ppm}: 43.3$ (2C), 43.5 (2C), 69.3 (2C), $69.6(2 \mathrm{C}), 70.2(2 \mathrm{C}), 70.3$ (2C), 103.3 (2C), 103.6 (2C), 114.2 (2C), 118.5 (2C), $120.4(2 \mathrm{C})$ 125.1 (2C), 125.8 (2C), 127.8 (2C), 128.8 (2C), 128.9 (2C), 129.2 (2C), 136.4 (2C), 146.3 (2C), 146.6 (2C), two quaternary carbon atoms were not assigned.

$N, N^{\prime}-\left(3,3^{\prime}-\left(2,2^{\prime}\right.\right.$-Oxybis (ethane-2,1-diyl)bis(oxy))bis(propane-3,1-diyl))bis(7-bromonaphthalene-2-amine), $4 \boldsymbol{b}$. Obtained from $220 \mathrm{mg}$ of trioxadiamine 1b. Eluent $\mathrm{CH}_{2} \mathrm{Cl}_{2} / \mathrm{MeOH} 200: 1$ Yield $235 \mathrm{mg}$ (37\%), yellowish glassy compound. (MALDI-TOF) found: 628.21. $\mathrm{C}_{30} \mathrm{H}_{34} \mathrm{Br}_{2} \mathrm{~N}_{2} \mathrm{O}_{3}$ requires $628.09[\mathrm{M}]^{+} .{ }^{1} \mathrm{H}$ NMR $\left(\mathrm{CDCl}_{3}, 298 \mathrm{~K}\right) \delta_{\mathrm{H}}$ ppm: $1.92\left(4 \mathrm{H}\right.$, quintet, $\left.{ }^{3} \mathrm{~J}=5.9 \mathrm{~Hz}\right), 3.29(4 \mathrm{H}, \mathrm{t}$, $\left.{ }^{3} J=6.2 \mathrm{~Hz}\right), 3.62\left(4 \mathrm{H}, \mathrm{t},{ }^{3} J=5.5 \mathrm{~Hz}\right), 3.61-3.65(4 \mathrm{H}, \mathrm{m}), 3.67-3.72$ (4H, m), 4.37 (2H, br.s), $6.62(2 \mathrm{H}$, br.s $), 6.81\left(2 \mathrm{H}, \mathrm{d},{ }^{3} J=8.8 \mathrm{~Hz}\right)$, $7.21\left(2 \mathrm{H}, \mathrm{d},{ }^{3} J=8.6 \mathrm{~Hz}\right), 7.47\left(2 \mathrm{H}, \mathrm{d},{ }^{3} J=8.6 \mathrm{~Hz}\right), 7.51\left(2 \mathrm{H}, \mathrm{d},{ }^{3} J\right.$ $=8.9 \mathrm{~Hz}), 7.72\left(2 \mathrm{H}\right.$, br.s). ${ }^{13} \mathrm{C} \mathrm{NMR}\left(\mathrm{CDCl}_{3}, 298 \mathrm{~K}\right) \delta_{\mathrm{c}}$ ppm: 28.8 (2C), $41.8(2 \mathrm{C}), 69.9$ (2C), 70.3 (2C), 70.7 (2C), $102.7^{\mathrm{C}}(2 \mathrm{C}), 118.5$ 
(2C), $120.4(2 \mathrm{C}), 124.8$ (2C), $125.6(2 \mathrm{C}), 127.7$ (2C), 128.7 (2C), 129.3 (2C), $136.6(2 \mathrm{C}), 147.0(2 \mathrm{C})$.

$N, N, N$, '-Tris(7-bromonaphth-2-yl)substituted trioxadiamine $\boldsymbol{8} \boldsymbol{b}$ was obtained as the second product in the synthesis of compound 4b. Eluent $\mathrm{CH}_{2} \mathrm{Cl}_{2} / \mathrm{MeOH}$ 200:1. Yield $67 \mathrm{mg}$ (10\%), yellowish glassy compound. (MALDI-TOF) found: 832.13. $\mathrm{C}_{40} \mathrm{H}_{39} \mathrm{Br}_{3} \mathrm{~N}_{2} \mathrm{O}_{3}$ requires $832.05[\mathrm{M}]^{+} .{ }^{1} \mathrm{H} \mathrm{NMR}\left(\mathrm{CDCl}_{3}, 298 \mathrm{~K}\right) \delta_{\mathrm{H}} \mathrm{ppm}: 1.91(2 \mathrm{H}$, quintet, $\left.{ }^{3} J=6.0 \mathrm{~Hz}\right), 1.99\left(2 \mathrm{H}\right.$, quintet, $\left.{ }^{3} J=6.3 \mathrm{~Hz}\right), 3.28(2 \mathrm{H}$, t, $\left.{ }^{3} J=6.3 \mathrm{~Hz}\right), 3.55\left(2 \mathrm{H}, \mathrm{t},{ }^{3} J=5.7 \mathrm{~Hz}\right), 3.63\left(2 \mathrm{H}, \mathrm{t},{ }^{3} J=5.6 \mathrm{~Hz}\right)$, $3.63-3.66(4 \mathrm{H}, \mathrm{m}), 3.71-3.75(4 \mathrm{H}, \mathrm{m}), 4.05\left(2 \mathrm{H}, \mathrm{t},{ }^{3} \mathrm{~J}=6.9 \mathrm{~Hz}\right)$, $4.39(1 \mathrm{H}$, br.s $), 6.61\left(1 \mathrm{H}, \mathrm{d},{ }^{4} J=1.9 \mathrm{~Hz}\right), 6.81\left(1 \mathrm{H}, \mathrm{dd},{ }^{3} J=8.8 \mathrm{~Hz}\right.$, $\left.{ }^{4} J=2.1 \mathrm{~Hz}\right), 7.21\left(1 \mathrm{H}, \mathrm{dd},{ }^{3} J=8.7 \mathrm{~Hz},{ }^{4} J=2.0 \mathrm{~Hz}\right), 7.24\left(2 \mathrm{H}, \mathrm{dd},{ }^{3} J\right.$ $\left.=8.7 \mathrm{~Hz},{ }^{4} \mathrm{~J}=2.1 \mathrm{~Hz}\right), 7.31\left(2 \mathrm{H}, \mathrm{d},{ }^{4} J=1.9 \mathrm{~Hz}\right), 7.39\left(2 \mathrm{H}, \mathrm{dd},{ }^{3} \mathrm{~J}=\right.$ $\left.8.7 \mathrm{~Hz},{ }^{4} J=1.8 \mathrm{~Hz}\right), 7.46\left(1 \mathrm{H}, \mathrm{d},{ }^{3} J=8.6 \mathrm{~Hz}\right), 7.50\left(1 \mathrm{H}, \mathrm{d},{ }^{3} J=8.8\right.$ $\mathrm{Hz}), 7.58\left(2 \mathrm{H}, \mathrm{d},{ }^{3} \mathrm{~J}=8.5 \mathrm{~Hz}\right), 7.64\left(2 \mathrm{H}, \mathrm{d},{ }^{3} \mathrm{~J}=9.0 \mathrm{~Hz}\right), 7.72(1 \mathrm{H}, \mathrm{d}$, $\left.{ }^{4} J=1.5 \mathrm{~Hz}\right), 7.83\left(2 \mathrm{H}\right.$, br.s). ${ }^{13} \mathrm{C} \mathrm{NMR}\left(\mathrm{CDCl}_{3}, 298 \mathrm{~K}\right) \delta_{\mathrm{c}} \mathrm{ppm}: 27.5$ (1C), 28.8 (1C), 41.7 (1C), 49.1 (1C), 68.2 (1C), 69.8 (1C), 70.3 (2C), 70.7 (2C), 102.6 (1C), 115.3 (2C), 118.4 (1C), 120.4 (3C), 122.8 (2C), 124.7 (1C), 125.5 (1C), 127.2 (2C), 127.6 (1C), 127.7 (2C), 128.6 (1C), 128.7 (2C), 128.8 (2C), 129.1 (2C), 129.2 (1C), 135.8 (2C), 136.5 (1C), 146.0 (2C), 146.9 (1C).

Oligomer $\mathbf{9 b}$ was obtained as the third product in the synthesis of compound $\mathbf{4 b}$. Eluent $\mathrm{CH}_{2} \mathrm{Cl}_{2} / \mathrm{MeOH} 100: 1$. Yield $29 \mathrm{mg}(6$ $\%$ ), yellowish glassy compound. (MALDI-TOF) found: 972.44 . $\mathrm{C}_{50} \mathrm{H}_{62} \mathrm{Br}_{2} \mathrm{~N}_{4} \mathrm{O}_{6}$ requires $972.30[\mathrm{M}]^{+} .{ }^{1} \mathrm{H}$ NMR $\left(\mathrm{CDCl}_{3}, 298 \mathrm{~K}\right) \delta_{\mathrm{H}}$ ppm: 1.91 (4H, quintet, $\left.{ }^{3} J=5.7 \mathrm{~Hz}\right), 1.92\left(4 \mathrm{H}\right.$, quintet, $\left.{ }^{3} J=5.8 \mathrm{~Hz}\right)$, $3.27\left(4 \mathrm{H}, \mathrm{t},{ }^{3} \mathrm{~J}=5.8 \mathrm{~Hz}\right), 3.28\left(4 \mathrm{H}, \mathrm{t},{ }^{3} \mathrm{~J}=6.2 \mathrm{~Hz}\right), 3.61\left(8 \mathrm{H}, \mathrm{t},{ }^{3} \mathrm{~J}=\right.$ 5.7), 3.61-3.65 (8H, m), 3.66-3.71 (8H, m), 4.33 (4H, br.s), 6.57 $\left(2 \mathrm{H}, \mathrm{dd},{ }^{3} J=8.7 \mathrm{~Hz},{ }^{4} J=2.0 \mathrm{~Hz}\right), 6.58(2 \mathrm{H}$, br.s $), 6.61$ (2H, br.s), $6.81\left(2 \mathrm{H}, \mathrm{dd},{ }^{3} J=8.7 \mathrm{~Hz},{ }^{4} J=2.1 \mathrm{~Hz}\right), 7.20\left(2 \mathrm{H}, \mathrm{dd},{ }^{3} J=8.4 \mathrm{~Hz}\right.$, $\left.{ }^{4} J=1,8 \mathrm{~Hz}\right), 7.40\left(2 \mathrm{H}, \mathrm{d},{ }^{3} J=8.6 \mathrm{~Hz}\right), 7.46\left(2 \mathrm{H}, \mathrm{d},{ }^{3} J=8.6 \mathrm{~Hz}\right)$, $7.51\left(2 \mathrm{H}, \mathrm{d},{ }^{3} J=8.9 \mathrm{~Hz}\right), 7.72\left(2 \mathrm{H}\right.$, br.s). ${ }^{13} \mathrm{C} \mathrm{NMR}\left(\mathrm{CDCl}_{3}, 298 \mathrm{~K}\right)$ $\delta_{\mathrm{c}}$ ppm: $28.8(2 \mathrm{C}), 29.0(2 \mathrm{C}), 41.7(4 \mathrm{C}), 69.8(4 \mathrm{C}), 70.2(4 \mathrm{C}), 70.6$ (4C), 102.6 (2C), 102.9 (2C), 113.8 (2C), 118.4 (2C), 120.3 (2C), 121.2 (1C), 124.7 (2C), 125.5 (2C), 127.6 (2C), $128.6(2 \mathrm{C}), 128.7$ (2C), 129.2 (2C), 136.6 (2C), 137.0 (1C), 146.7 (2C), 147.0 (2C).

$N^{1}, N^{I^{\prime}}$-(Ethane-1,2-diyl)bis( $N^{3}$-(7-bromonaphthalene-2-yl) propane-1,3-diamine), $4 c$. Obtained from $174 \mathrm{mg}$ of tetraamine 1c. Eluent $\mathrm{CH}_{2} \mathrm{Cl}_{2} / \mathrm{MeOH} / \mathrm{NH}_{3}$ aq 100:20:1. Yield $158 \mathrm{mg}(27 \%)$, yellowish glassy compound. (MALDI-TOF) found: 583.1025. $\mathrm{C}_{28} \mathrm{H}_{33} \mathrm{Br}_{2} \mathrm{~N}_{4}$ requires $583.1072[\mathrm{M}+\mathrm{H}]^{+}$. ${ }^{1} \mathrm{H} \mathrm{NMR}\left(\mathrm{CDCl}_{3}, 298 \mathrm{~K}\right)$ $\delta_{\mathrm{H}}$ ppm: $1.79\left(4 \mathrm{H}\right.$, quintet, $\left.{ }^{3} \mathrm{~J}=6.5 \mathrm{~Hz}\right), 1.89(2 \mathrm{H}, \mathrm{br} . \mathrm{s}), 2.72(4 \mathrm{H}$, s), $2.73\left(4 \mathrm{H}, \mathrm{t},{ }^{3} J=6.6 \mathrm{~Hz}\right), 3.21\left(4 \mathrm{H}, \mathrm{t},{ }^{3} J=6.5 \mathrm{~Hz}\right), 4.50$ (2H, br.s), $6.60\left(2 \mathrm{H}, \mathrm{d},{ }^{4} J=1.5 \mathrm{~Hz}\right), 6.80\left(2 \mathrm{H}, \mathrm{dd},{ }^{3} J=8.7 \mathrm{~Hz},{ }^{4} J=2.2 \mathrm{~Hz}\right)$, $7.22\left(2 \mathrm{H}, \mathrm{dd},{ }^{3} J=8.6 \mathrm{~Hz},{ }^{4} J=1.8 \mathrm{~Hz}\right), 7.46\left(2 \mathrm{H}, \mathrm{d},{ }^{3} J=8.6 \mathrm{~Hz}\right)$, $7.51\left(2 \mathrm{H}, \mathrm{d},{ }^{3} \mathrm{~J}=8.8 \mathrm{~Hz}\right), 7.73(2 \mathrm{H}$, br.s $) .{ }^{13} \mathrm{C} \mathrm{NMR}\left(\mathrm{CDCl}_{3}, 298\right.$ K) $\delta_{c}$ ppm: 29.0 (2C), $42.4(2 \mathrm{C}), 48.0(2 \mathrm{C}), 49.3$ (2C), $102.6(2 \mathrm{C})$, 118.2 (2C), 120.3 (2C), 124.7 (2C), 125.5 (2C), 127.6 (2C), 128.6 (2C), 129.2 (2C), $136.5(2 \mathrm{C}), 146.8(2 \mathrm{C})$.

Oligomer $9 c$ was obtained as the second product in the synthesis of compound 4c. Eluent $\mathrm{CH}_{2} \mathrm{Cl}_{2} / \mathrm{MeOH} / \mathrm{NH}_{3}$ aq 100:20:2. Yield $166 \mathrm{mg}$ (37\%), yellowish glassy compound. (MALDI-TOF) found: $881.3156 . \mathrm{C}_{46} \mathrm{H}_{59} \mathrm{Br}_{2} \mathrm{~N}_{8}$ requires $881.3229[\mathrm{M}+\mathrm{H}]^{+} .{ }^{1} \mathrm{H} \mathrm{NMR}$ $\left(\mathrm{CDCl}_{3}, 298 \mathrm{~K}\right) \delta_{\mathrm{H}} \mathrm{ppm}: 1.76\left(4 \mathrm{H}\right.$, quintet $\left.{ }^{3} J=6.3 \mathrm{~Hz}\right), 1.77(4 \mathrm{H}$, quintet, $\left.{ }^{3} J=6.0 \mathrm{~Hz}\right), 2.64-2.73(16 \mathrm{H}, \mathrm{m}), 3.15\left(4 \mathrm{H}, \mathrm{t},{ }^{3} J=6.5 \mathrm{~Hz}\right)$, $3.19\left(4 \mathrm{H}, \mathrm{t},{ }^{3} \mathrm{~J}=6.3 \mathrm{~Hz}\right), 4.48$ (4H, br.s), 6.54-6.60 (6H, m), 6.76$6.81(2 \mathrm{H}, \mathrm{m}), 7.18-7.22(2 \mathrm{H}, \mathrm{m}), 7.37-7.50(6 \mathrm{H}, \mathrm{m}), 7.72$ (2H, br.s), $\mathrm{NH}$ protons of dialkylamino groups were not assigned. ${ }^{13} \mathrm{C} \mathrm{NMR}$ $\left(\mathrm{CDCl}_{3}, 298 \mathrm{~K}\right) \delta_{\mathrm{c}} \mathrm{ppm}: 28.8(2 \mathrm{C}), 28.9(2 \mathrm{C}), 42.3(2 \mathrm{C}), 42.4(2 \mathrm{C})$, 47.8 (2C), 47.9 (2C), 49.0 (4C), 102.5 (2C), 102.8 (2C), 113.7 (2C), 118.3 (2C), 120.2 (2C), 121.2 (1C), 124.6 (2C), 125.4 (2C), 127.5 (2C), 128.5 (2C), 128.6 (2C), 129.2 (2C), 136.5 (2C), 136.9 (1C), 146.6 (2C), 146.8 (2C).

Typical procedure for the synthesis of cyclodimers 7 from compounds 4.

A two-neck flask equipped with a condenser and magnetic stirrer, flushed with dry argon, was charged with compound 4a-c, $\mathrm{Pd}(\mathrm{dba})_{2}(8 \mathrm{~mol} \%)$, BINAP $(9 \mathrm{~mol} \%)$ and dioxane to make $0.02 \mathrm{M}$ solution, the mixture was stirred for 2-3 min, then equimolar amount of appropriate polyamine $\mathbf{1 a - c}$ and ${ }^{t} \mathrm{BuONa}$ ( 3 equiv.) were added, and the reaction mixture was refluxed for 6-10 h. After cooling it down to ambient temperature the reaction mixture was diluted with $\mathrm{CH}_{2} \mathrm{Cl}_{2}$, the residue was filtered off, the organic solvents were evaporated in vacuo, and the residue was chromatographed on silica gel using a sequence of eluents: $\mathrm{CH}_{2} \mathrm{Cl}_{2}, \mathrm{CH}_{2} \mathrm{Cl}_{2} / \mathrm{MeOH}$ (200:1-3:1), $\mathrm{CH}_{2} \mathrm{Cl}_{2} / \mathrm{MeOH} / \mathrm{NH}_{3}$ aq (100:20:1-10:4:1).

Cyclic dimer $7 \boldsymbol{a}$. Obtained from compound $4 \mathbf{a}(70 \mathrm{mg}, 0.13$ $\mathrm{mmol})$, dioxadiamine 1a $(19 \mathrm{mg}, 0.13 \mathrm{mmol})$, in the presence of $\mathrm{Pd}(\mathrm{dba})_{2}(6 \mathrm{mg}, 0.01 \mathrm{mmol})$, BINAP $(7.3 \mathrm{mg}, 0.012 \mathrm{mmol}),{ }^{t} \mathrm{BuONa}$ (37 mg, $0.39 \mathrm{mmol}$ ), in $6.5 \mathrm{ml}$ dioxane. Eluent $\mathrm{CH}_{2} \mathrm{Cl}_{2} / \mathrm{MeOH} 75: 1$. Yield $8 \mathrm{mg}(11 \%)$, yellowish glassy compound. (MALDI-TOF) found: 544.3018. $\mathrm{C}_{32} \mathrm{H}_{40} \mathrm{~N}_{4} \mathrm{O}_{4}$ requires $544.3050[\mathrm{M}]^{+}$. ${ }^{1} \mathrm{H}$ NMR $\left(\mathrm{CDCl}_{3}, 298 \mathrm{~K}\right) \delta_{\mathrm{H}}$ ppm: $3.28\left(8 \mathrm{H}, \mathrm{t},{ }^{3} J=5.2 \mathrm{~Hz}\right), 3.66(8 \mathrm{H}, \mathrm{s})$, $3.70\left(8 \mathrm{H}, \mathrm{t},{ }^{3} J=5.1 \mathrm{~Hz}\right), 4.15$ (4H, br.s), $6.44\left(4 \mathrm{H}, \mathrm{d},{ }^{4} J=2.1 \mathrm{~Hz}\right)$, $6.64\left(4 \mathrm{H}, \mathrm{dd},{ }^{3} \mathrm{~J}=8.8 \mathrm{~Hz},{ }^{4} \mathrm{~J}=2.1 \mathrm{~Hz}\right), 7.41\left(4 \mathrm{H}, \mathrm{d},{ }^{3} J=8.7 \mathrm{~Hz}\right)$. ${ }^{13} \mathrm{C} \mathrm{NMR}\left(\mathrm{CDCl}_{3}, 298 \mathrm{~K}\right) \delta_{\mathrm{c}} \mathrm{ppm}: 43.6(4 \mathrm{C}), 69.5(4 \mathrm{C}), 70.2(4 \mathrm{C})$, 104.5 (4C), 113.3 (4C), 121.7 (2C), 128.7 (4C), 136.8 (2C), 146.3 (4C).

Cyclic dimer $\mathbf{7 b}$. Obtained from compound $\mathbf{4 b}$ (148 mg, 0.24 $\mathrm{mmol})$, trioxadiamine $\mathbf{1 b}(52 \mathrm{mg}, 0.24 \mathrm{mmol})$, in the presence of Pd(dba) (11 mg, $0.019 \mathrm{mmol})$, BINAP (13 mg, $0.021 \mathrm{mmol}$ ), ${ }^{t} \mathrm{BuONa}(70 \mathrm{mg}, 0.72 \mathrm{mmol})$, in $12 \mathrm{ml}$ dioxane. Eluent $\mathrm{CH}_{2} \mathrm{Cl}_{2} /$ $\mathrm{MeOH}$ 100:1-50:1. Yield $45 \mathrm{mg}$ (28\%), yellowish glassy compound. (MALDI-TOF) found: 688.41. $\mathrm{C}_{40} \mathrm{H}_{56} \mathrm{~N}_{4} \mathrm{O}_{6}$ requires $688.42[\mathrm{M}]^{+}$ ${ }^{1} \mathrm{H}$ NMR $\left(\mathrm{CDCl}_{3}, 298 \mathrm{~K}\right) \delta_{\mathrm{H}} \mathrm{ppm}: 1.88\left(8 \mathrm{H}\right.$, quintet, $\left.{ }^{3} J=6.0 \mathrm{~Hz}\right)$, $3.27\left(8 \mathrm{H}, \mathrm{t},{ }^{3} \mathrm{~J}=6.4 \mathrm{~Hz}\right), 3.59\left(8 \mathrm{H}, \mathrm{t},{ }^{3} \mathrm{~J}=5.8 \mathrm{~Hz}\right), 3.59-3.62(8 \mathrm{H}$, m), 3.66-3.70 (8H, m), 4.12 (4H, br.s), $6.51\left(4 \mathrm{H}, \mathrm{d},{ }^{4} J=1.9 \mathrm{~Hz}\right)$, $6.57\left(4 \mathrm{H}, \mathrm{dd},{ }^{3} J=8.6 \mathrm{~Hz},{ }^{4} \mathrm{~J}=2.0 \mathrm{~Hz}\right), 7.39\left(4 \mathrm{H}, \mathrm{d},{ }^{3} J=8.7 \mathrm{~Hz}\right) .{ }^{13} \mathrm{C}$ NMR ( $\left.\mathrm{CDCl}_{3}, 298 \mathrm{~K}\right) \delta_{\mathrm{c}} \mathrm{ppm}: 29.1(4 \mathrm{C}), 41.7(4 \mathrm{C}), 69.6(4 \mathrm{C}), 70.3$ (4C), 70.7 (4C), 103.3 (4C), 113.6 (4C), 121.3 (2C), 128.5 (4C), $137.0(2 \mathrm{C}), 146.7$ (4C).

A mixture of cyclic tetramer and hexamer $\mathbf{1 0 b}(n=3,5)$ was obtained as by-product in the synthesis of the cyclic dimer $\mathbf{7 b}$. Eluent $\mathrm{CH}_{2} \mathrm{Cl}_{2} / \mathrm{MeOH} 10: 1$. Yield $18 \mathrm{mg}$ (11\%), yellowish glassy compound. (MALDI-TOF) found: 1376.75. $\mathrm{C}_{80} \mathrm{H}_{112} \mathrm{~N}_{8} \mathrm{O}_{12}$ requires 1376.84 [M] $]^{+}$for $10 \mathrm{~b}(\mathrm{n}=3)$. found: 2065.09. $\mathrm{C}_{120} \mathrm{H}_{168} \mathrm{~N}_{12} \mathrm{O}_{18}$ requires 2065.26 $[\mathrm{M}]^{+}$for $10 \mathrm{~b}(\mathrm{n}=5)$. ${ }^{1} \mathrm{H}$ NMR $\left(\mathrm{CDCl}_{3}, 298 \mathrm{~K}\right) \delta_{\mathrm{H}} \mathrm{ppm}$ : $1.89(4(\mathrm{n}+1) \mathrm{H}$, br.s $), 3.26\left(4(\mathrm{n}+1) \mathrm{H}, \mathrm{t},{ }^{3} J=5.7 \mathrm{~Hz}\right), 3.59(8(\mathrm{n}+1)$ H, br.s $), 3.66(4(\mathrm{n}+1) \mathrm{H}$, br.s $), 6.54\left(2(\mathrm{n}+1) \mathrm{H}, \mathrm{d},{ }^{3} J=9.3 \mathrm{~Hz}\right), 6.57$ $(2(\mathrm{n}+1) \mathrm{H}$, br.s $), 7.38\left(2(\mathrm{n}+1) \mathrm{H}, \mathrm{d},{ }^{3} J=9.3 \mathrm{~Hz}\right), \mathrm{NH}$ protons were not assigned. ${ }^{13} \mathrm{C}$ NMR $\left(\mathrm{CDCl}_{3}, 298 \mathrm{~K}\right) \delta_{\mathrm{c}} \mathrm{ppm}: 29.1(2(\mathrm{n}+1) \mathrm{C})$, $41.7(2(n+1) C), 69.8(2(n+1) C), 70.2(2(n+1) C), 70.6(2(n+1) C)$, $102.9(2(n+1) C), 113.8(2(n+1) C), 118.1((n+1) C), 128.5(2(n+1)$ C), $137.0((\mathrm{n}+1) \mathrm{C}), 146.7(2(\mathrm{n}+1) \mathrm{C})$.

Cyclic dimer $7 \boldsymbol{c}$. Obtained from compound $4 \mathbf{c}(158 \mathrm{mg}$, $0.3 \mathrm{mmol})$, tetraamine $1 \mathrm{c}(52 \mathrm{mg}, 0.3 \mathrm{mmol})$, in the presence of $\mathrm{Pd}(\mathrm{dba})_{2}$ (14 mg, $\left.0.024 \mathrm{mmol}\right)$, BINAP (17 mg, $0.027 \mathrm{mmol}$ ), ${ }^{t} \mathrm{BuONa}(86 \mathrm{mg}, 0.9 \mathrm{mmol})$, in $15 \mathrm{ml}$ dioxane. Eluent $\mathrm{CH}_{2} \mathrm{Cl}_{2}$ / $\mathrm{MeOH} / \mathrm{NH}_{3}$ aq 100:20:3. Yield $37 \mathrm{mg}$ (21\%), yellowish glassy compound. (MALDI-TOF) found: 596.4221. $\mathrm{C}_{36} \mathrm{H}_{52} \mathrm{~N}_{8}$ requires $596.4314[\mathrm{M}]^{+} .{ }^{1} \mathrm{H} \mathrm{NMR}\left(\mathrm{CDCl}_{3}, 298 \mathrm{~K}\right) \delta_{\mathrm{H}} \mathrm{ppm}: 1.73$ (8H, quintet, $\left.{ }^{3} J=6.2 \mathrm{~Hz}\right), 2.69\left(8 \mathrm{H}, \mathrm{t},{ }^{3} \mathrm{~J}=6.4 \mathrm{~Hz}\right), 2.71(8 \mathrm{H}, \mathrm{s}), 3.19\left(8 \mathrm{H}, \mathrm{t},{ }^{3} \mathrm{~J}=\right.$ $6.4 \mathrm{~Hz}), 6.52-6.58(8 \mathrm{H}, \mathrm{m}), 7.38\left(4 \mathrm{H}, \mathrm{d},{ }^{3} J=9.0 \mathrm{~Hz}\right), \mathrm{NH}$ protons were not assigned. ${ }^{13} \mathrm{C} \mathrm{NMR}\left(\mathrm{CDCl}_{3}, 298 \mathrm{~K}\right) \delta_{\mathrm{c}} \mathrm{ppm}$ : 29.3 (4C), 42.7 (4C), 47.8 (4C), 48.9 (4C), 103.3 (4C), 113.5 (4C), 121.3 (2C), 128.6 (4C), $137.0(2 \mathrm{C}), 146.7(4 \mathrm{C})$.

Cyclic tetramer $10 \mathbf{c}(\mathrm{n}=3)$ was obtained as the second product in the synthesis of the cyclic dimer 7c. Eluent $\mathrm{CH}_{2} \mathrm{Cl}_{2} / \mathrm{MeOH} / \mathrm{NH}_{3}$ aq 10:4:1. Yield $19 \mathrm{mg}(11 \%)$, yellowish glassy compound. (MALDITOF) found: 1192.83. $\mathrm{C}_{72} \mathrm{H}_{104} \mathrm{~N}_{16}$ requires $1192.86[\mathrm{M}]^{+} .{ }^{1} \mathrm{H}$ NMR (DMSO- $\left.d_{6}, 298 \mathrm{~K}\right) \delta_{\mathrm{H}}$ ppm: 1.74 (16H, br.s), 2.69 (32H, br.s), 3.08 (16H, br.s), 5.59 (8H, br.s), 6.41 (8H, br.s), 6.53 (8H, d, ${ }^{3} J=8.1$ $\mathrm{Hz}), 7.26\left(8 \mathrm{H}, \mathrm{d},{ }^{3} J=8.1 \mathrm{~Hz}\right)$, NH protons of dialkylamino groups were not assigned. ${ }^{13} \mathrm{C}$ NMR (DMSO- $\left.d_{6}, 298 \mathrm{~K}\right) \delta_{\mathrm{c}} \mathrm{ppm:} 28.0(8 \mathrm{C})$, 41.0 (8C), 46.7 (8C), 47.5 (8C), 101.1 (8C), 113.1 (8C), 119.7 (4C), 127.9 (8C), $137.1(4 \mathrm{C}), 146.9(8 \mathrm{C})$. 
Cyclic dimer $7 \boldsymbol{a b}$. Obtained from compound $\mathbf{4 b}$ (105 mg, 0.17 $\mathrm{mmol})$, dioxadiamine 1a $(25 \mathrm{mg}, 0.17 \mathrm{mmol})$, in the presence of $\mathrm{Pd}(\mathrm{dba}),(8 \mathrm{mg}, 0.014 \mathrm{mmol})$, BINAP ( $9 \mathrm{mg}, 0.014 \mathrm{mmol}),{ }^{t} \mathrm{BuONa}$ (49 mg, $0.51 \mathrm{mmol}$ ), in $8 \mathrm{ml}$ dioxane. Eluent $\mathrm{CH}_{2} \mathrm{Cl}_{2} / \mathrm{MeOH} 50: 1$. Yield $14 \mathrm{mg}$ (13\%), yellowish glassy compound. (MALDI-TOF) found: 616.50. $\mathrm{C}_{36} \mathrm{H}_{48} \mathrm{~N}_{4} \mathrm{O}_{5}$ requires 616.36 [M] ${ }^{+} .{ }^{1} \mathrm{H} \mathrm{NMR}\left(\mathrm{CDCl}_{3}\right.$, $298 \mathrm{~K}) \delta_{\mathrm{H}}$ ppm: $1.91\left(4 \mathrm{H}\right.$, quintet, $\left.{ }^{3} J=6.0 \mathrm{~Hz}\right), 3.26-3.40(8 \mathrm{H}, \mathrm{m})$, 3.57-3.75 (20H, m), 4.13 (4H, br.s), 6.59-6.65 (8H, m), $7.40(2 \mathrm{H}$, d, $\left.{ }^{3} \mathrm{~J}=8.4 \mathrm{~Hz}\right), 7.42\left(2 \mathrm{H}, \mathrm{d},{ }^{3} \mathrm{~J}=8.7 \mathrm{~Hz}\right) .{ }^{13} \mathrm{C} \mathrm{NMR}\left(\mathrm{CDCl}_{3}, 298\right.$ K) $\delta_{\mathrm{c}}$ ppm: $29.0(2 \mathrm{C}), 41.7(2 \mathrm{C}), 43.7(2 \mathrm{C}), 69.5(2 \mathrm{C}), 69.8(2 \mathrm{C})$, 70.2 (4C), 70.6 (2C), 102.9 (2C), 103.5 (2C), 113.9 (2C), 114.0 (2C), 121.6 (2C), 128.5 (2C), 128.6 (2C), 136.9 (2C), 146.4 (2C), $146.7(2 \mathrm{C})$

Typical procedure for the synthesis of cyclic dimers 7 via bis(polyamine) derivatives 5 .

A two-neck flask equipped with a condenser and magnetic stirrer, flushed with argon, was charged with 2,7-dibromonaphthalene (143 mg, $0.5 \mathrm{mmol}), \mathrm{Pd}(\mathrm{dba})_{2}$ (11.5 mg, $0.02 \mathrm{mmol}, 4 \mathrm{~mol} \%$ ), diphosphine ligand (15 mg of Xantphos or $14 \mathrm{mg}$ of BINAP, 0.023 $\mathrm{mmol}, 4.5 \mathrm{~mol} \%), 5 \mathrm{ml}$ of dioxane. The mixture was stirred for 2-3 min, then appropriate polyamine 1 a-c $(2 \mathrm{mmol})$ and ${ }^{t} \mathrm{BuONa}(144$ $\mathrm{mg}, 1.5 \mathrm{mmol}$ ) were added and the reaction mixture was refluxed for 4-6 h. After cooling it down to ambient temperature an aliquot $(0.5 \mathrm{ml})$ was taken and analyzed using NMR and MALDI-TOF spectroscopy. Then 2,7-dibromonaphthalene (386 mg, $1.35 \mathrm{mmol}$ ), $\mathrm{Pd}(\mathrm{dba})_{2}(62 \mathrm{mg}, 0.11 \mathrm{mmol}, 8 \mathrm{~mol} \%$ ), BINAP (76 mg, $0.12 \mathrm{mmol}$, $9 \mathrm{~mol} \%), 20 \mathrm{ml}$ abs. dioxane and ${ }^{t} \mathrm{BuONa}(390 \mathrm{mg}, 4 \mathrm{mmol})$ were added, and the reaction mixture was refluxed for $6-15 \mathrm{~h}$. After cooling it down to ambient temperature the reaction mixture was diluted with $\mathrm{CH}_{2} \mathrm{Cl}_{2}$, the residue was filtered off, the organic solvents were evaporated in vacuo, and the residue was chromatographed on silica gel using a sequence of eluents: $\mathrm{CH}_{2} \mathrm{Cl}_{2}, \mathrm{CH}_{2} \mathrm{Cl}_{2} / \mathrm{MeOH}$ (200:1-3:1), $\mathrm{CH}_{2} \mathrm{Cl}_{2} / \mathrm{MeOH} / \mathrm{NH}_{3}$ aq (100:20:1-10:4:1).

$N^{2}, N^{7}$-Bis(2-(2-(2-aminoethoxy)ethoxy)ethyl)naphthalene2,7-diamine, 5a. Obtained in situ using Xantphos ligand and 296 mg of dioxadiamine 1a. (MALDI-TOF) found: 421.32. $\mathrm{C}_{22} \mathrm{H}_{37} \mathrm{~N}_{4} \mathrm{O}_{4}$ requires $421.28[\mathrm{M}+\mathrm{H}]^{+} .{ }^{1} \mathrm{H} \mathrm{NMR}\left(\mathrm{CDCl}_{3}, 298 \mathrm{~K}\right) \delta_{\mathrm{H}} \mathrm{ppm}: 2.83(4 \mathrm{H}$, $\left.\mathrm{t},{ }^{3} \mathrm{~J}=5.2 \mathrm{~Hz}\right), 3.35\left(4 \mathrm{H}, \mathrm{t},{ }^{3} \mathrm{~J}=4.9 \mathrm{~Hz}\right), 3.47\left(4 \mathrm{H}, \mathrm{t},{ }^{3} J=5.2 \mathrm{~Hz}\right), 3.59-$ $3.63(8 \mathrm{H}, \mathrm{m}), 3.72\left(4 \mathrm{H}, \mathrm{t},{ }^{3} J=5.3 \mathrm{~Hz}\right), 4.23(2 \mathrm{H}, \mathrm{br} . \mathrm{s}), 6.58-6.61(4 \mathrm{H}$, m), $7.41\left(2 \mathrm{H}, \mathrm{d},{ }^{3} J=9.3 \mathrm{~Hz}\right) .{ }^{13} \mathrm{C} \mathrm{NMR}\left(\mathrm{CDCl}_{3}, 298 \mathrm{~K}\right) \delta_{\mathrm{c}} \mathrm{ppm}: 41.7$ (2C), 43.4 (2C), 69.5 (2C), 70.2 (4C), 73.3 (2C), 103.3 (2C), 114.1 (2C), 121.6 (1C), 128.6 (2C), 136.7 (1C), 146.3 (2C).

Cyclic dimer $7 \boldsymbol{a}$ was synthesized from in situ obtained compound 5a. Eluent $\mathrm{CH}_{2} \mathrm{Cl}_{2} / \mathrm{MeOH}$ 75:1. Yield $26 \mathrm{mg}$ (10\%). Spectral data are given above. A mixture of cyclic trimer and tetramer $7 \mathbf{a}(\mathrm{n}=2,3)$ was obtained as the side product. Eluent $\mathrm{CH}_{2} \mathrm{Cl}_{2} / \mathrm{MeOH}$ 50:1-20:1. Yield $58 \mathrm{mg}$ (21\%). (MALDI-TOF) found: 816.41. $\mathrm{C}_{48} \mathrm{H}_{60} \mathrm{~N}_{6} \mathrm{O}_{6}$ requires $816.46[\mathrm{M}]^{+}$for $7 \mathbf{a}(\mathrm{n}=2)$. found: 1088.67. $\mathrm{C}_{64} \mathrm{H}_{80} \mathrm{~N}_{8} \mathrm{O}_{8}$ requires $1088.60[\mathrm{M}]^{+}$for $7 \mathbf{a}(\mathrm{n}=3)$. ${ }^{1} \mathrm{H}$ NMR $\left(\mathrm{CDCl}_{3}, 298 \mathrm{~K}\right) \delta_{\mathrm{H}} \mathrm{ppm}: 3.30-3.40(4(\mathrm{n}+1) \mathrm{H}, \mathrm{m}), 3.60-$ $3.75(8(\mathrm{n}+1) \mathrm{H}, \mathrm{m}), 6.56-6.64(4(\mathrm{n}+1) \mathrm{H}, \mathrm{m}), 7.32-7.46(2(\mathrm{n}+1)$ $\mathrm{H}, \mathrm{m}) .{ }^{13} \mathrm{C} \mathrm{NMR}\left(\mathrm{CDCl}_{3}, 298 \mathrm{~K}\right) \delta_{\mathrm{c}} \mathrm{ppm}: 43.4(2(\mathrm{n}+1) \mathrm{C}), 69.4+$ $69.5(2(n+1) C), 70.1(2(n+1) C), 103.4(2(n+1) C), 114.0(2(n+1) C)$, $128.7(2(n+1) C), 146.3(2(n+1) C), 2(n+1)$ quaternary carbon atoms were not assigned.

$N^{2}, N^{7}$-Bis(3-(2-(2-(3-aminopropoxy)ethoxy)ethoxy)propyl)naphthalene-2,7-diamine, $5 \boldsymbol{b}$. Obtained in situ using Xantphos ligand and $440 \mathrm{mg}$ of trioxadiamine 1b. (MALDI-TOF) found: 565.44. $\mathrm{C}_{30} \mathrm{H}_{53} \mathrm{~N}_{4} \mathrm{O}_{6}$ requires $565.40[\mathrm{M}+\mathrm{H}]^{+} .{ }^{1} \mathrm{H} \mathrm{NMR}\left(\mathrm{CDCl}_{3}, 298\right.$ K) $\delta_{\mathrm{H}}$ ppm: $1.63\left(4 \mathrm{H}\right.$, quintet, $\left.{ }^{3} \mathrm{~J}=6.5 \mathrm{~Hz}\right), 1.86\left(4 \mathrm{H}\right.$, quintet, ${ }^{3} \mathrm{~J}=$ $6.1 \mathrm{~Hz}), 2.68\left(4 \mathrm{H}, \mathrm{t},{ }^{3} J=6.8 \mathrm{~Hz}\right), 3.22\left(4 \mathrm{H}, \mathrm{t},{ }^{3} \mathrm{~J}=6.5 \mathrm{~Hz}\right), 3.46(4 \mathrm{H}$, $\left.\mathrm{t},{ }^{3} J=6.2 \mathrm{~Hz}\right), 3.47-3.58(20 \mathrm{H}, \mathrm{m}), 6.48-6.52(4 \mathrm{H}, \mathrm{m}), 7.32(2 \mathrm{H}$, d, $\left.{ }^{3} J=8.3 \mathrm{~Hz}\right) .{ }^{13} \mathrm{C}$ NMR $\left(\mathrm{CDCl}_{3}, 298 \mathrm{~K}\right) \delta_{\mathrm{c}} \mathrm{ppm}: 28.8(2 \mathrm{C}), 33.1$ (2C), 39.2 (2C), $41.5(2 \mathrm{C}), 69.3$ (2C), 69.6 (4C), 70.0 (2C), 70.1 (2C), 70.4 (2C), 102.7 (2C), 113.6 (2C), 121.0 (1C), 128.3 (2C), 136.8 (1C), 146.5 (2C).

Cyclic dimer $7 \boldsymbol{b}$ was synthesized from in situ obtained compound 5b. Eluent $\mathrm{CH}_{2} \mathrm{Cl}_{2} / \mathrm{MeOH}$ 50:1. Yield $104 \mathrm{mg}$ (30\%).
Spectral data are given above. Macrocycle $6 \mathrm{~b}$ was obtained as the side product. Eluent $\mathrm{CH}_{2} \mathrm{Cl}_{2} / \mathrm{MeOH} 200: 1$. Yield $100 \mathrm{mg}$ (24\%). Spectral data are given in ref. ${ }^{[14]} \mathrm{A}$ mixture of cyclic trimer, tetramer and pentamer $10 \mathbf{b}(n=2-4)$ was obtained as the side product. Eluent $\mathrm{CH}_{2} \mathrm{Cl}_{2} / \mathrm{MeOH}$ 20:1. Yield $90 \mathrm{mg}$ (25\%). (MALDI-TOF) found: 1032.55. $\mathrm{C}_{60} \mathrm{H}_{84} \mathrm{~N}_{6} \mathrm{O}_{9}$ requires $1032.63[\mathrm{M}]^{+}$for $\mathbf{1 0 b}(\mathrm{n}=2)$. found: 1376.95. $\mathrm{C}_{80} \mathrm{H}_{112} \mathrm{~N}_{8} \mathrm{O}_{12}$ requires $1376.84[\mathrm{M}]^{+}$for $\mathbf{1 0 b}(\mathrm{n}=3)$. found: 1721.12. $\mathrm{C}_{100} \mathrm{H}_{140} \mathrm{~N}_{10} \mathrm{O}_{15}$ requires $1721.05[\mathrm{M}]^{+}$for $\mathbf{1 0 b}(\mathrm{n}=4) .{ }^{1} \mathrm{H}$ NMR $\left(\mathrm{CDCl}_{3}, 298 \mathrm{~K}\right) \delta_{\mathrm{H}} \mathrm{ppm}$ : $1.91\left(4(\mathrm{n}+1) \mathrm{H}\right.$, quintet, ${ }^{3} J=6.1$ $\mathrm{Hz}), 3.27\left(\mathrm{t},{ }^{3} J=5.9 \mathrm{~Hz}\right)+3.28\left(\mathrm{t},{ }^{3} J=6.2 \mathrm{~Hz}\right)(4(\mathrm{n}+1) \mathrm{H}), 3.56-$ $3.62(8(\mathrm{n}+1) \mathrm{H}, \mathrm{m}), 3.66-3.69(4(\mathrm{n}+1) \mathrm{H}, \mathrm{m}), 4.00(2(\mathrm{n}+1) \mathrm{H}, \mathrm{br.s})$, 6.53-6.61 $(4(\mathrm{n}+1) \mathrm{H}, \mathrm{m}), 7.40\left(\mathrm{~d},{ }^{3} J=8.0 \mathrm{~Hz}\right)+7.41\left(\mathrm{~d},{ }^{3} J=8.5\right.$ $\mathrm{Hz})(2(\mathrm{n}+1) \mathrm{H}) .{ }^{13} \mathrm{C} \mathrm{NMR}\left(\mathrm{CDCl}_{3}, 298 \mathrm{~K}\right) \delta_{\mathrm{c}} \mathrm{ppm}: 29.0(2(\mathrm{n}+1) \mathrm{C})$, $41.6(2(n+1) C), 69.7(2(n+1) C), 70.1(2(n+1) C), 70.5(2(n+1) C)$, $102.8(2(\mathrm{n}+1) \mathrm{C}), 113.7(2(\mathrm{n}+1) \mathrm{C}), 121.2((\mathrm{n}+1) \mathrm{C}), 128.4(2(\mathrm{n}+1)$ C), $137.0((\mathrm{n}+1) \mathrm{C}), 146.6(2(\mathrm{n}+1) \mathrm{C})$.

$N^{1}, N^{I^{\prime}}$-(Naphthalene-2,7-diyl)bis ( $N^{3}$-(3-(3-aminopropylamino)propyl)propane-1,3-diamine), 5d. Obtained in situ using BINAP ligand and $376 \mathrm{mg}$ of tetraamine 1d. (MALDI-TOF) found: 501.41. $\mathrm{C}_{28} \mathrm{H}_{53} \mathrm{~N}_{8}$ requires $501.44[\mathrm{M}+\mathrm{H}]^{+} .{ }^{1} \mathrm{H} \mathrm{NMR}\left(\mathrm{CDCl}_{3}, 298\right.$ K) $\delta_{\mathrm{H}}$ ppm: $1.52-1.63(8 \mathrm{H}, \mathrm{m}), 1.77\left(4 \mathrm{H}\right.$, quintet, $\left.{ }^{3} \mathrm{~J}=6.6 \mathrm{~Hz}\right)$, 2.55-2.70 (20H, m), $3.19\left(4 \mathrm{H}, \mathrm{t},{ }^{3} J=6.4 \mathrm{~Hz}\right), 6.49-6.55(4 \mathrm{H}, \mathrm{m})$, $7.35\left(2 \mathrm{H}, \mathrm{d},{ }^{3} J=8.6 \mathrm{~Hz}\right) \cdot{ }^{13} \mathrm{C}$ NMR $\left(\mathrm{CDCl}_{3}, 298 \mathrm{~K}\right) \delta_{\mathrm{c}} \mathrm{ppm}: 29.2$ (2C), 30.2 (2C), 33.6 (2C), 40.2 (2C), 42.7 (2C), 47.7 (4C), 48.4 (4C), 102.8 (2C), $113.6(2 \mathrm{C}), 121.1$ (1C), 128.4 (2C), 136.9 (1C), $146.6(2 \mathrm{C})$.

Cyclic dimer $7 \boldsymbol{d}$ was synthesized from in situ obtained compound 5d. Eluent $\mathrm{CH}_{2} \mathrm{Cl}_{2} / \mathrm{MeOH} / \mathrm{NH}_{3}$ aq 100:25:5, yellow glassy compound. Yield $75 \mathrm{mg}$ (22\%). (MALDI-TOF) found: 625.4662. $\mathrm{C}_{38} \mathrm{H}_{57} \mathrm{~N}_{8}$ requires 625.4706 $[\mathrm{M}+\mathrm{H}]^{+}$. ' $\mathrm{H} \mathrm{NMR}\left(\mathrm{CDCl}_{3}\right.$, $298 \mathrm{~K}) \delta_{\mathrm{H}}$ ppm: $1.67\left(4 \mathrm{H}\right.$, quintet, $\left.{ }^{3} J=5.7 \mathrm{~Hz}\right), 1.74(8 \mathrm{H}$, quintet, $\left.{ }^{3} J=6.3 \mathrm{~Hz}\right), 2.64-2.72(16 \mathrm{H}, \mathrm{m}), 3.15\left(8 \mathrm{H}, \mathrm{t},{ }^{3} J=6.3 \mathrm{~Hz}\right), 4.14$ (4H, br.s), 6.52-6.56 (8H, m), $7.38\left(4 \mathrm{H}, \mathrm{d},{ }^{3} J=9.1 \mathrm{~Hz}\right), \mathrm{NH}$ protons of dialkylamino groups were not assigned. ${ }^{13} \mathrm{C} \mathrm{NMR}\left(\mathrm{CDCl}_{3}, 298\right.$ K) $\delta_{\text {c }}$ ppm: 29.0 (6C), 42.7 (4C), 48.1 (4C), 48.9 (4C), 103.1 (4C), 113.6 (4C), 121.2 (2C), 128.5 (4C), 137.0 (2C), 146.7 (4C). Macrocycle $6 \mathrm{~d}$ was obtained as the side product. Eluent $\mathrm{CH}_{2} \mathrm{Cl}_{2} /$ $\mathrm{MeOH} / \mathrm{NH}_{3}$ aq 100:25:5. Yield $65 \mathrm{mg}(19 \%)$. Spectral data are given in ref. ${ }^{14}$ Cyclic trimer $\mathbf{1 0 d}(\mathrm{n}=2)$ was obtained as the side product. Eluent $\mathrm{CH}_{2} \mathrm{Cl}_{2} / \mathrm{MeOH} / \mathrm{NH}_{3}$ aq $10: 4: 1$. Yield $80 \mathrm{mg}$ (24 $\%$ ), yellow glassy compound. (MALDI-TOF) found: 937.68. $\mathrm{C}_{57} \mathrm{H}_{85} \mathrm{~N}_{12}$ requires $937.70[\mathrm{M}+\mathrm{H}]^{+} .{ }^{1} \mathrm{H} \mathrm{NMR}\left(\mathrm{CDCl}_{3}, 298 \mathrm{~K}\right) \delta_{\mathrm{H}}$ ppm: $1.55-1.82(18 \mathrm{H}, \mathrm{m}), 2.56-2.74(24 \mathrm{H}, \mathrm{m}), 3.18\left(12 \mathrm{H}, \mathrm{t},{ }^{3} \mathrm{~J}=\right.$ $6.1 \mathrm{~Hz}), 4.24$ (6H, br.s), $6.52\left(6 \mathrm{H}, \mathrm{d},{ }^{3} J=8.5 \mathrm{~Hz}\right), 6.57$ (6H, br.s), $7.38\left(6 \mathrm{H}, \mathrm{d},{ }^{3} \mathrm{~J}=8.5 \mathrm{~Hz}\right), \mathrm{NH}$ protons of dialkylamino groups were not assigned. ${ }^{13} \mathrm{C} \mathrm{NMR}\left(\mathrm{CDCl}_{3}, 298 \mathrm{~K}\right) \delta_{\mathrm{c}} \mathrm{ppm:} 29.2(6 \mathrm{C})$, 30.1 (2C), 42.7 (6C), 48.4 (12C), 102.9 (6C), 113.7 (6C), 128.5 (6C), $137.0(3 \mathrm{C}), 146.7$ (6C), three quaternary carbon atoms were not assigned.

\section{Results and Discussion}

Our synthetic aproach to the nitrogen- and oxygencontaining macrocycles based on 2,7-diaminonaphthalene is represented in Scheme 1. To synthesize the macrocycles 6 bearing the naphthalene fragment in the cycle, two successive Pd-catalyzed amination reactions of 2,7-dibromonaphtalene with a linear polyamine molecule should be carried out, which proceed in a one-pot manner when the synthesis of the macrocycles 6 is the synthetic goal (Scheme 1). The first amination reaction affords a linear intermediate compounds 3 whose intramolecular macrocyclization leads to the target compounds $\mathbf{6}$. The cyclization competes with the formation of linear compounds: $N, N^{\prime}$-bis(7-bromonaphth2-yl) substituted polyamines 4 and 2,7-bis(polyamine) 
A. D. Averin et al.<smiles>[X]CNCCC(C)C(C)C</smiles>
2<smiles>[X]CNc1ccc2ccc(Br)cc2c1</smiles><smiles>NCCOCCOCCN</smiles><smiles>NCCCOCCOCCOCCCN</smiles><smiles>NCCCNCCNCCCN</smiles><smiles>NCCCNCCCNCCCN</smiles><smiles>[X]CCNc1ccc2ccc(NCC[X])cc2c1</smiles><smiles>[X]CCNc1ccc2ccc(NCCCCCCCCCC)cc2c1</smiles>

6<smiles>[X]CNc1ccc2ccc(NCCNc3ccc4ccc(NCC)cc4c3)cc2c1</smiles>

7

Scheme 1.

substituted naphthalenes 5. By changing catalytic system and reaction conditions, we have shown how to manage this process and achieve good yields of the macrocycles $\mathbf{6}^{\left[{ }^{[14]}\right.}$ The best yields of the macrocycles were obtained when the starting compounds were reacted in the stoichiometric ratio. Interestingly, cyclodimers 7 were often isolated in notable yields under these conditions. These large macrocycles represent a new family of macrocyclic ligands and are of potential interest for supramolecular studies. Thus we focused on the development of the catalytic conditions for the synthesis of these large macrocycles. According to Scheme 1, target cyclodimers 7 can be formed from either intermediate $\mathbf{4}$ or intermediate $\mathbf{5}$, and herein we compare these two synthetic approaches with the objective to find an optimal synthetic route to these ligands.

According to the first synthetic route, $N, N^{\prime}$-bis(7bromonaphth-2-yl) substituted polyamines 4a-c were synthesized using the $\mathrm{Pd}$-catalyzed reaction of 1 equiv. of di- and polyamines 1a-c with 2.2 equiv. of 2,7dibromonaphthalene (2) (Scheme 2).

The reactivity of oxadiamines $\mathbf{1 a}, \mathbf{b}$ and polyamine $\mathbf{1 c}$ was found to be significantly different. At first we carried out the cross-coupling reaction using a catalytic system $\mathrm{Pd}(\mathrm{dba})_{2}$ /BINAP (BINAP = 2,2'-bis(diphenylphosphino)1,1'-binaphthalene) which was found to be efficient in the synthesis of $N, N^{\prime}$-bis(haloaryl) substituted di-

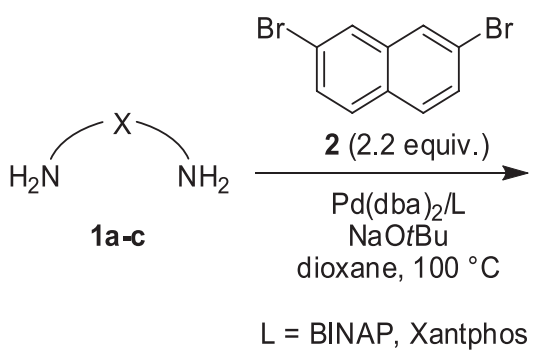

$\mathrm{L}=\mathrm{BINAP}$, Xantphos

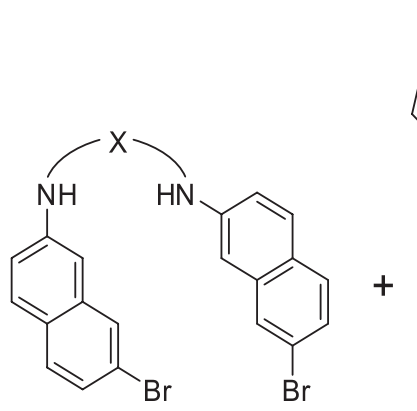

$4 a, 27 \% ; 4 b, 37 \% ; 4 c, 27 \%$

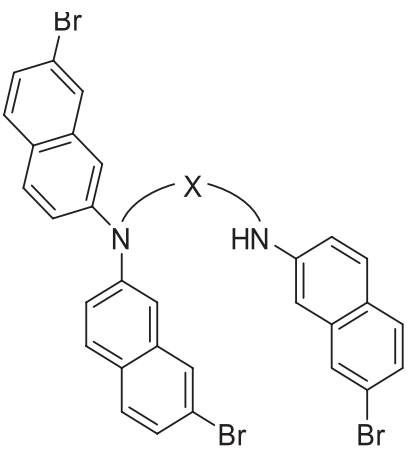

$8 \mathrm{a}, 10 \%$;

$\mathbf{8 b}, 10 \%$<smiles>[X]CCNc1ccc2ccc(NCCNc3ccc4ccc(Br)cc4c3)cc2c1</smiles>

$9 a, 13 \% ; 9 b, 6 \% ; 9 c, 37 \%$

Scheme 2. 


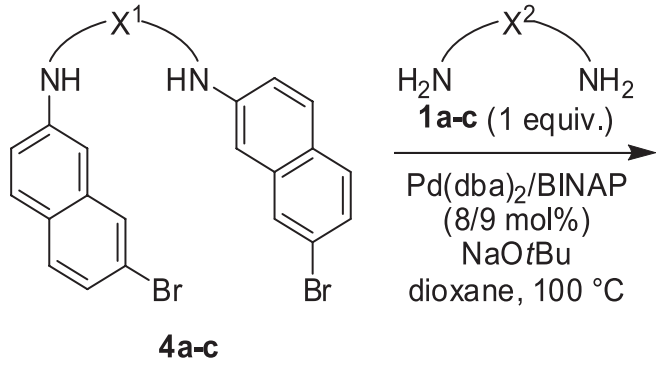

Scheme 3.

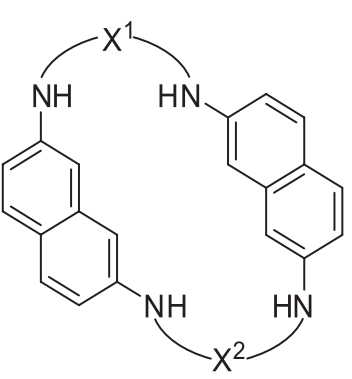

$7 a, 11 \% ; 7 b, 28 \%$;

$7 c, 21 \% ; 7 a b, 13 \%$

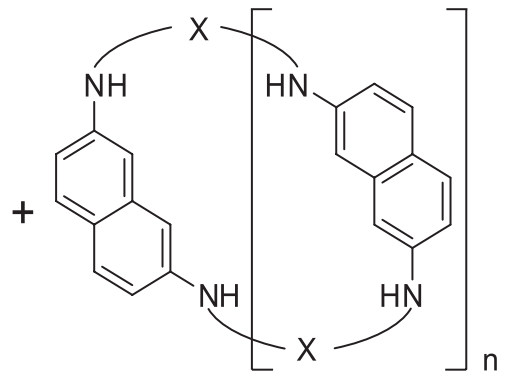

$10 \mathrm{~b}(\mathrm{n}=3,5), 11 \%$ $10 \mathrm{c}(\mathrm{n}=3), 11 \%$
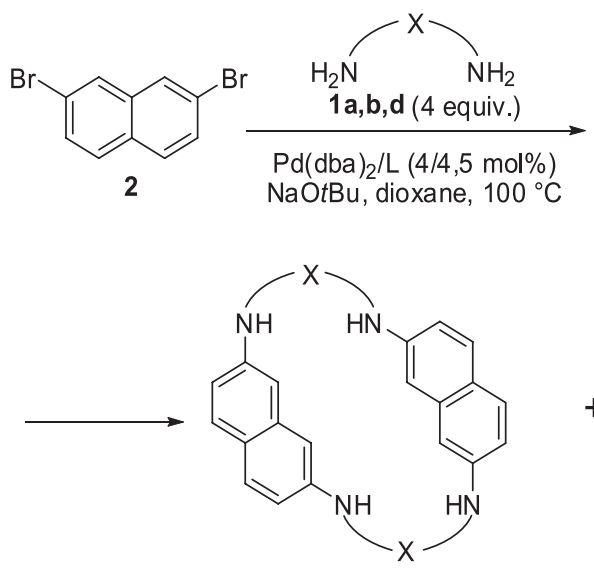

$7 \mathrm{a}, 10 \%$

$7 \mathbf{7 b}, 30 \%$<smiles>[X]CNc1ccc2ccc(NCCN)cc2c1</smiles>

$\mathbf{5 a , b , d}$ in situ

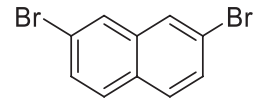

$\underset{\mathrm{Pd}(\mathrm{dba})_{2} / \mathrm{BINAP}(8 / 9 \mathrm{~mol} \%)}{\mathrm{NaO} \text { tBu, dioxane }, 100^{\circ} \mathrm{C}}$
$\mathrm{Na}$.

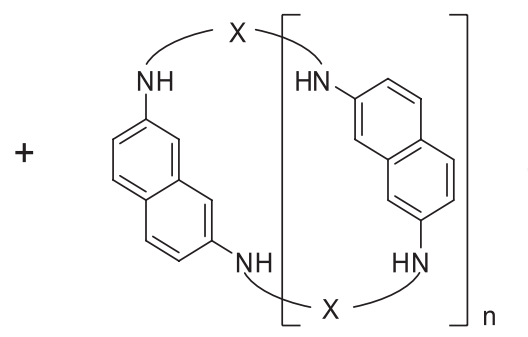

$10 \mathbf{a}(\mathrm{n}=2,3), 21 \%$ $10 b(n=2-4) .25 \%$

Scheme 4.

and polyamines. ${ }^{[17]}$ However, in the case of dioxa- and trioxadiamines $\mathbf{1 a}, \mathbf{b}$, side products like $N, N$-diarylated diamines $\mathbf{8 a}, \mathbf{b}$ and linear oligomers $\mathbf{9 a}, \mathbf{b}$ were predominant in the reaction mixtures, and the target compounds $\mathbf{4 a}, \mathbf{b}$ could not be isolated in pure form. We changed BINAP for a less efficient ligand Xantphos (9,9-dimethyl-4,5bis(diphenylphosphino)xanthene) and diluted the reaction mixtures (0.05 M solutions instead of usual 0.1-0.2 M were used). As a result, we managed to isolate target diarylated oxadiamines $\mathbf{4 a}, \mathbf{b}$ in moderate yields together with $N, N, N$ 'triarylated amines $\mathbf{8 a}, \mathbf{b}$ and more complex oligomers 9a,b resulting from the diamination reaction. The $N, N$ 'diarylation of the tetraamine 1c should be conducted using BINAP as ligand because Xantphos was not efficient for this reaction. Under these conditions, the target product $\mathbf{4 c}$ and compound $9 \mathbf{c}$ were obtained as major products and were isolated in pure form by column chromatorgphy.

The $N, N^{\prime}$-bis(bromonaphthyl) derivatives 4a-c were introduced in the Pd-catalyzed macrocyclization reaction with the same polyamines 1a-c (Scheme 3). In this case we applied $8 \mathrm{~mol} \%$ of the catalytic system $\mathrm{Pd}(\mathrm{dba})_{2} / \mathrm{BINAP}$, equimolar amounts of starting compounds and $0.02 \mathrm{M}$ solutions of the reactants in dioxane. The best yield of the cyclodimers 7 was obtained for trioxadiamine derivative $\mathbf{7 b}$ $(28 \%)$, tetraamine $1 \mathrm{c}$ provided $21 \%$ yield of the compound 7c, while the short dioxadiamine 1a gave a poor yield of the corresponding cyclodimer $7 \mathbf{a}(11 \%)$. The unsymmetric macrocycle $\mathbf{7 a}, \mathbf{b}$ was obtained upon reacting compound $\mathbf{4 b}$ with dioxadiamine 1a. Cyclic tetramers and hexamers 10b,c were also isolated as by-products in these reactions.

An alternative method for the synthesis of cyclodimers included the formation of 2,7-bis(polyamine)substituted naphthalenes $\mathbf{5 a , b , d}$ which cannot be separated by the column chromatography in pure form and were used in situ (Scheme 4). The reactions were conducted using 4 equiv. of di- and polyamines $\mathbf{1 a}, \mathbf{b}, \mathbf{d}$ in dioxane $(0.1 \mathrm{M}$ solutions $)$ in the presence of $4 \mathrm{~mol} \%$ of the catalyst. As in the synthesis of $N, N$ 'bis(bromonaphthyl) substituted polyamines 4, Xantphos was applied in the reactions with dioxa- and trioxadiamines $\mathbf{1 a , b}$ and BINAP was used for tetraamine 1d. After NMR analysis proving that compounds $\mathbf{5 a}, \mathbf{b}, \mathbf{d}$ were the major products in the reaction mixtures, 3 equiv. of 2,7-dibromonaphthalene (2), $8 \mathrm{~mol} \%$ of $\mathrm{Pd}(\mathrm{dba})_{2} / \mathrm{BINAP}$ catalytic system, additional amounts of ${ }^{t} \mathrm{BuONa}$ and dioxane were added and the reaction mixtures were refluxed for $24 \mathrm{~h}$. The best yield of the cyclodimer was obtained in the case of trioxadiamine (7b, $30 \%$ ), compound $\mathbf{7 d}$ was isolated in $22 \%$ yield, while the product 7a containing two dioxadiamine chains again was obtained in a low yield $(10 \%)$. Cyclic oligomers of higher masses $\mathbf{1 0 a}, \mathbf{b}, \mathbf{d}$ were isolated in comparable yields $21-25 \%$ in all cases, and also we observed the formation of the macrocycles comprising one naphthalene and one 
polyamine unit $\mathbf{6 b}, \mathbf{d}$. The latter compounds were formed at the second step of the process due to the presence of the excess of polyamines $\mathbf{1}$ in the reaction mixture which were not consumed at the first step.

\section{Conclusions}

To sum up, we investigated two alternative approaches for the synthesis of macrocycles comprising two naphthalene and two polyamine moieties (cyclodimers) based on the Pd-catalyzed amination reaction. The first one, including the synthesis of $N, N^{\prime}$-bis(7-bromonaphth-2-yl) substituted polyamines, needs chromatographic isolation of these intermediate compounds due to the formation of by-products. The second route is a one-pot method with the formation of 2,7-bis(polyamine) substituted naphthalenes which are used in situ in the second macrocyclization step. Two synthetic routes were compared for different target cyclodimers. Optimization of experimental conditions are needed in both synthetic routes and the nature of the catalyst is a key factor influencing the product yields. The optimization of the onepot reaction seems to be easier and better overall yields of the target cyclic dimers were obtained. Xantphos ligand was shown to be efficient for the synthesis of naphthalene linear derivatives with oxadiamines, while BINAP was found to be useful in the analogous reactions with tetraamine and in all macrocyclization reactions. Careful optimization of catalytic conditions allows to achieve the overall product yields up to $30 \%$ without employing multistep methodologies.

Acknowledgements. This work was carried out in the frame of the International Associated French-Russian Laboratory of Macrocycle Systems and Related Materials (LAMREM) of the Centre National de la Recherche Scientifique (CNRS). It was financially supported by CNRS, RFBR grant 12-0393107, and by the Russian Academy of Sciences program
P-8 "Development of the methods for the synthesis of new chemicals and creation of new materials".

\section{References}

1. Lüttringhaus A. Liebigs Ann. Chem. 1937, 528, 181-210.

2. Gallant A.J., Yun M., Sauer M., Yeung C.S., MacLachlan M.J. Org. Lett. 2005, 7, 4827-4830.

3. Sharghi H., Zare A. Synthesis 2006, 999-1004.

4. Khoshbin M.S., Ovchinnikov M.V., Khalid S., Mirkin C.A., Stern C., Zakharov L.N., Rheingold A.L. Chem. Asian J. 2006, 1, 686-692.

5. Eshgi H., Mirzaei M., Mehdi E., Shahry H. J. Chem. Res. 2007, 272-274

6. Patra G.K., Datta D. Ind. J. Chem., Sect. A 2000, 39, 480-483.

7. Rasadkina E.N., Slitikov P.V., Evdokimenkova Yu.B., Nifantyev E.E. Zh. Obshch. Khim. 2003, 73, 1279-1283.

8. Yamato T., Okabe R., Miyamoto S., Miyazaki M. J. Chem. Res. 2006, 593-595.

9. Tran H.-A., Ashram M., Mizyed S., Thompson D.W., Georghiou P.E. J. Inclusion Phenom. Macrocyclic Chem. 2008, 60, 4349.

10. Lukyanenko N.G., Lyapunov A.Yu., Kirichenko T.I., Botoshansky M.M., Simonov Yu.A., Fonari M.S. Tetrahedron Lett. 2005, 46, 2109-2112.

11. Kieran A.L., Pascu S.I., Jarosson T., Maxwell J., Sanders J.K. M. Chem. Commun. 2005, 1842-1844.

12. Qin H., He Y., Qing G., Hu C., Yang X. Tetrahedron: Assymetry 2006, 17, 2143-2148.

13. Alfonso I., Burguete M. I., Galindo F., Luis S.V., Vigara L. J. Org. Chem. 2007, 72, 7947-7956.

14. Averin A.D., Uglov A.N., Beletskaya I.P. Chem. Lett. 2008, 37, 1074-1075.

15. Neenan T.X., Whitesides G.M. J. Org. Chem. 1988, 53, 24892496.

16. Ukai T., Kawazura H., Ishii Y., Bonnet J.J., Ibers J.A. J. Organomet. Chem. 1974, 65, 253-266.

17. Uglov A.N., Averin A.D., Buryak A.K., Beletskaya I.P. ARKIVOC 2011, viii, 99-122. 